\title{
Boosting Active Contours for Weld Pool Visual Tracking in Automatic Arc Welding
}

Liu, Jinchao; Fan, Zhun; Olsen, Søren Ingvor; Christensen, Kim Hardam; Kristensen, Jens Klastrup

Published in:

I E E E Transactions on Automation Science and Engineering

Link to article, DOI:

10.1109/TASE.2015.2498929

Publication date:

2015

Document Version

Publisher's PDF, also known as Version of record

Link back to DTU Orbit

Citation (APA):

Liu, J., Fan, Z., Olsen, S. I., Christensen, K. H., \& Kristensen, J. K. (2015). Boosting Active Contours for Weld Pool Visual Tracking in Automatic Arc Welding. I E E E Transactions on Automation Science and Engineering, 99, 1-13. https://doi.org/10.1109/TASE.2015.2498929

\section{General rights}

Copyright and moral rights for the publications made accessible in the public portal are retained by the authors and/or other copyright owners and it is a condition of accessing publications that users recognise and abide by the legal requirements associated with these rights.

- Users may download and print one copy of any publication from the public portal for the purpose of private study or research.

- You may not further distribute the material or use it for any profit-making activity or commercial gain

- You may freely distribute the URL identifying the publication in the public portal 


\title{
Boosting Active Contours for Weld Pool Visual Tracking in Automatic Arc Welding
}

\author{
Jinchao Liu, Zhun Fan, Senior Member, IEEE, \\ Søren Ingvor Olsen, Kim Hardam Christensen, and Jens Klæstrup Kristensen
}

\begin{abstract}
Detecting the shape of the non-rigid molten metal during welding, so-called weld pool visual sensing, is one of the central tasks for automating arc welding processes. It is challenging due to the strong interference of the high-intensity arc light and spatters as well as the lack of robust approaches to detect and represent the shape of the nonrigid weld pool. We propose a solution using active contours including an prior for the weld pool boundary composition. Also, we apply Adaboost to select a small set of features that captures the relevant information. The proposed method is applied to weld pool tracking and the presented results verified its feasibility.
\end{abstract}

Note to Practitioners-Welding quality is highly dependent on the human welder's experiences and skills in the gas tungsten arc welding process. In particular, detecting the shape of the nonrigid molten metal during welding plays an important role in improving the welding quality since it provides a prime feedback mechanism for welding control. However, this is a challenging task due to a number of reasons, e.g., strong interference and changing working environment. Applying classical image processing algorithms directly, such as active contour models $(\mathrm{ACM})$, cannot guarantee satisfactory performance due to the above-mentioned reasons. Actually, in practice, ACM more often than not failed to capture the welding pool boundary. In this work, we integrate an applicationdependent prior to the definition of the energy functional of a traditional ACM, thus allowing us to use machine learning techniques to improve the performance of traditional ACM. In density estimation of the prior, AdaBoost is utilized to select features that are most suitable for Gaussian Bayesian classifiers to differentiate wanted boundaries and unwanted ones (e.g., spurious boundaries caused by interfering noise). The proposed approach improves the performance of the traditional ACM and captures the boundary of the

Manuscript received July 28, 2015; accepted August 21, 2015. This paper was recommended for publication by Associate Editor K. Fregene and Editor M. Wang upon evaluation of the reviewers' comments. This work was supported in part by FORCE Technology and the Danish Agency for Science Technology and Innovation.

J. Liu was with the Department of Mechanical Engineering, Technical University of Denmark, 2800 Kgs. Lyngby, Denmark, and also with Welding and Production Innovation, FORCE Technology. DK-2605 Brødby, Denmark. He is now with VisionMetric Ltd. CT2 7NJ Canterbury, Kent, U.K. (e-mail: liujinchao2000@gmail.com).

Z. Fan was with the Department of Mechanical Engineering, Technical University of Denmark, $2800 \mathrm{Kgs}$. Lyngby, Denmark. He is now with Guangdong Provincial Key Laboratory of Digital Signal and Image Processing, College of Engineering, Shantou University. 515063, Shantou, China (e-mail: zfan@stu. edu.cn).

S. I. Olsen is with the Department of Computer Science, University of Copenhagen, 2100 Copenhagen, Denmark(e-mail: ingvor@diku.dk).

K. H. Christensen is with Welding and Production Innovation, FORCE Technology, 2605 Brødby, Denmark (e-mail: kmc@force.dk).

J. K. Kristensen was with Welding and Production Innovation, FORCE Technology, 2605 Brødby, Denmark (e-mail: jens@klaestrup.dk).

Color versions of one or more of the figures in this paper are available online at http://ieeexplore.ieee.org.

Digital Object Identifier 10.1109/TASE.2015.2498929 welding pool successfully. It can also be generalized to other applications that need to integrate application-dependent priors to enhance classical methods, which may otherwise be not applicable in real-world implementations.

Index Terms-Active contours, AdaBoost, machine vision, welding automation, weld pool tracking.

\section{INTRODUCTION}

I $\mathrm{N}$ modern industry, welding plays an important role for joining materials, and arc welding has been the dominant process for many years. The majority of arc welding is still being made manually, and the need for automating the arc welding process has become more intensive. The productivity of the manual welding is low, and its quality varies significantly from person to person. Also, the by-products generated during the welding process such as airborne particles and gases, the highintensity light, and the heat are unhealthy for human welders.

Visual information on the weld pool is hard to acquire because of the interference of the extremely high-intensity arc light. Use of auxiliary light sources to illuminate the weld pool regions and to suppress the arc light has also been investigated ${ }^{1}$ [1], [2], [29]. Solutions using 2-D or 3-D structured light have been proposed in [33], [35]-[39], [51], and [54]-[56]. For instance, Liu et al. proposed to use a 3-D structure light weld pool sensing solution for real-time weld pool and penetration control and showed promising results. In [50], a real-time top-face vision system for controlling the weld pool size was proposed. In [57], Wu et al. proposed a weld pool monitoring solution using a low-cost CCD camera for constant-current gas tungsten arc welding. In [46], Shen et al. presents a passive vision system for both seam tracking and weld pool control in square-wave alternating current gas tungsten arc welding. Very recently, Shi et al. presents an innovative laser-vision-based sensing method to measure the weld pool oscillation frequency in GTAW-P in [47]. A detailed review of vision-based sensing may be found in [53].

In this paper, we present our work on developing a passive vision system which is only equipped with a single off-the-shelf CCD camera and optical filters yet capable of extracting relevant information, e.g., weld pool shapes, for closed-loop control and on-line quality inspection of the welding process.

As shown in Fig. 1(a), a typical frame of the weld pool captured during welding consists of several different regions, including the weld pool, arc light, welding wire, workpiece as well

${ }^{1}$ [Online]. Available: http://www.cavitar.com/technology/cavilux 


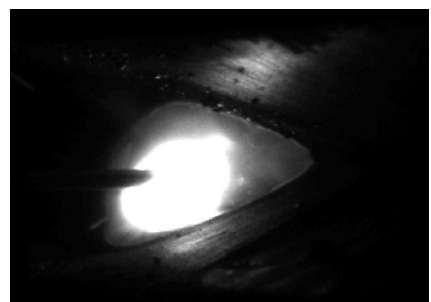

(a)

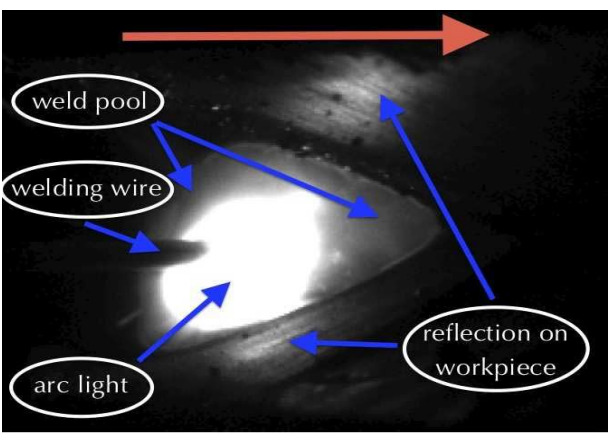

(c)

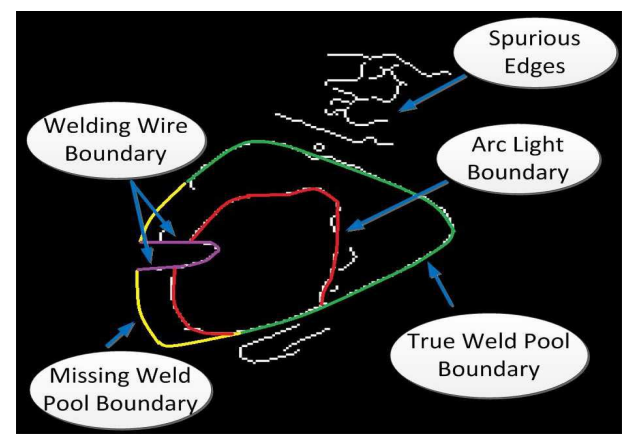

(d)

Fig. 1. Different edges in a typical weld pool image captured during welding. (a) A typical weld pool image. (b) The edges obtained by applying Canny edge detector. (c) Different regions appearing in the weld pool image. The red arrow indicates the welding direction. (d) Manually annotation of different edges. The true weld pool boundary is composed of the green curve and the yellow curve.

as some reflection of arc light on the workpiece, as illustrated in Fig. 1(c). Fig. 1(b) shows the edge image obtained by applying a Canny edge detector [8]. Different kinds of boundaries were marked in different colors, including a segment of the true weld pool boundary (marked in green curve), the arc light boundary (marked in red curve), the welding wire boundary (marked in purple curve), the missing weld pool boundary (marked in yellow curve), and the spurious edges. The true weld pool boundary is composed of the green and yellow curves. The arc light boundary marked in red curve should be considered as noise even though it is indeed a well-defined gradient-induced edge. Please note that a segment of the weld pool boundary marked in yellow escaped from the detection, because the missing segment is not well defined by gradient.

To identity the boundaries correctly, prior information is needed. In [45] a model-based approach for detecting liquid droplets in laser-enhanced gas metal arc welding(GMAW) is proposed. Here the droplet shape was used as a prior.

We propose a prior characterizing the different weld pool areas and the contours separating them. This will be used in an active contour setting and applied in weld pool boundary tracking. Our method applies a naive Bayesian classifier with a generative probabilistic model representing the application-dependent prior. Also, we employ a feature selective AdaBoost to select a subset of sufficient features to characterize the different boundary types. As a result, the speed of the active contours is greatly boosted.

The remainder of this article is organized as follows. In Section II, existing works related to active contours are discussed. In Section III, the framework of feature selective AdaBoost active contours is presented. In Section IV, feature selective Adaboost based active contour is applied to the real-world application of weld pool visual sensing in automatic arc welding. In Section $\mathrm{V}$, the experiments are presented and the results are discussed. Finally, a conclusion is made with some future work discussed in Section VI.

\section{RELATED WORKS}

Active contours, also referred to as deformable models, have been extensively studied for years and found numerous applications such as image segmentation, visual tracking [30], [58], [59] and many other areas. Active contours provide an elegant framework to incorporate prior knowledge.

Active contours may be formulated as a minimization problem of an energy functional. The basic framework is first introduced by Kass et al. in [30] as

$$
\begin{aligned}
& E(\mathcal{C}) \\
& =\int_{0}^{1}\left(\alpha\left\|\frac{d \mathcal{C}}{d s}\right\|^{2}+\beta\left\|\frac{d^{2} \mathcal{C}}{d s^{2}}\right\|^{2}+E_{\text {image }}+E_{\text {con }}+E_{\text {Prior }}\right) d s .
\end{aligned}
$$

The first two terms constitute so-called internal forces and are related to the characteristic of the curve itself. The effect of minimizing these two terms is to regulate or smooth the curve during evolution. The third term is an image dependent term which drives the curve to the desired boundary of the object. Often this term is defined as a function of the gradient magnitude such that the curve will be attracted to the high-gradient boundary in the image as follows:

$$
E_{\text {image }}(I)=g(\nabla I)
$$

where $g$ is a monotonically decreasing function and $I$ denotes the intensity of the image. The last two terms $E_{\text {con }}$ and $E_{\text {Prior }}$ of (1) represents the external constraint forces and the applicationdependent prior. We will later describe how these terms may be formulated.

Minimizing (1) may be challenging because it may have many local minima. Therefore, the solution may be sensitive to the initialization. In [19], [52] statistical optimizers like simulated annealing have been applied to improve convergence. Such methods are computationally heavy, yet they do not guarantee finding the global minimum. In [4], [17], [25], and [60], graph cut techniques are used to achieve a fast and global minimization of the energy functionals. The limitation here is that not all energy functionals are graph-representable, i.e., some of them cannot be minimized by graph cut [32]. 
The image-dependent energy term $E_{\text {image }}$ may be defined from the intensity gradient magnitude as in (2), but may also be defined by any other feature [12], [13]. One pioneer work is due to Chan and Vese in [13], where a region-based energy functional is proposed. Instead of modeling the edges (as sharp transition of intensity), it models different regions in terms of intensity and regards the boundary as the transition between different regions. Similar idea was also proposed in [62].

More recently, much effort has been made to improve the performance of region-based active contours. Basically there are two directions for improvement which deal with the limitations of the original Chan-Vese model. The first one is to employ more complicated and flexible probabilistic region models [3], [10], [20], [22], [28], [40]. Another direction is to use texture features [40], [42], [43] to detect the texture-induced edges.

Finally, boundary and region based techniques may be combined [9], [41], [43], [62]. This framework is particularly important in cases like the one shown in Fig. 1, when the desired boundary of the object is a mixture of different kinds of boundaries.

In addition to prior terms $E_{\text {Prior }}$ defined by intensity [40], [42], [62], texture, or other image features, priors may alternatively be defined by knowledge on the shape of the final contour [7], [14], [18], [24]. Such knowledge may be build from expert annotations and may include statistics on shape variability. This approach also is relevant when occlusion may occur [16], [23], [44], [61].

In this work, we propose to use Adaboost to select the features that best define the borders between the segments of the weld pool images. The next subsections briefly introduce the feature selective AdaBoost and discusses how to model the prior knowledge.

\section{Boosting Active Contours}

The AdaBoost algorithm was first proposed by Freund and Schapire [21]. The idea is that we can train a series of weak classifiers sequentially and combine them linearly to obtain a strong classifier. Since its invention, feature selective AdaBoost is one of the very successful variants which aims at performing dimension reduction in feature space when building probabilistic models [48].

In the original AdaBoost, all samples are represented in the same feature space. At each iteration, we change the weight of each individual sample using the associated training error so that the new weak classifier concentrates more on the samples that were misclassified in the previous step. In some cases, the dimension of the feature space where samples are situated in may be very high and this slows down the online classification process. It is therefore natural to consider performing dimension reduction, i.e., feature selection before or when building a probabilistic models. In the feature-selective AdaBoost, at each iteration, a new weak classifier is trained for each feature and the weak classifier which has the minimal weighted error is then selected. The feature that is associated with the optimal weak classifier may also be regarded as the best feature carrying discriminating information for the correction of the previous misclassification in the current step.
The two-class AdaBoost can be generalized to multi-class classifiers by means of one-versus-one strategy. For $k$-class classification, $k(k-1) / 2$ binary classifiers are trained and then combined [5].

Assume that there are a group of well-defined boundaries $\left\{B_{1}, B_{2}, \ldots, B_{K}\right\}$ in the image. Depending on the application, only a few of them are considered as the desired ones. In general, the model of the prior can take the form of

$$
p_{\text {app }}(\mathbf{x})= \begin{cases}P\left(\mathbf{x} \in B_{k}\right), & \text { if } \mathbf{x} \in B_{k} \text { and } B_{k} \in \mathcal{B}^{+} \\ 0, & \text { if } \mathbf{x} \in B_{k} \text { and } B_{k} \in \mathcal{B}^{-}\end{cases}
$$

where $\mathcal{B}^{+}=\bigcup_{k^{+}} B_{k^{+}}$stands for the union of all of the desired boundaries. $\mathcal{B}^{-}=\bigcup_{k^{-}} B_{k^{-}}$stands for the union of the remaining boundaries. The core idea of (3) is that a probabilistic model is learned for each class and that we penalize the undesired classes and keep the desired ones. Then the major task is to build the probabilistic model for each class by using supervised learning methods.

Often, the intensity or gradient alone does not contain sufficient information to separate the classes. More features are needed. On the other hand, using many features increases the processing time and make the corresponding probabilistic models complex. We propose to use feature selective AdaBoost to select a subset of the features and construct the probabilistic models only based on this subset.

There are two immediate mechanisms for constructing multi-class membership probabilities. The first mechanism is to couple the pairwise estimates of the binary classifiers [27]. The second scheme, which we have used in this work, is to construct a single Bayesian classifier based on the union of all selected features, i.e., $\bigcup_{k, l=1}^{K} F_{k l}$, instead of coupling all of the binary classifiers. More specifically, by using feature selective AdaBoost, we can obtain a set of crucial features

$$
\bigcup_{k, l=1}^{K} F_{k l}=\left\{f_{i}, i=1,2, \ldots, W\right\}
$$

and corresponding class-conditional distributions

$$
P\left(f_{i} \mid B_{k}\right), i=1,2, \ldots, W, k=1,2, \ldots, K
$$

where $W$ is the total number of the selected crucial features. We can establish a naive Bayes classifier as follows:

$$
\begin{aligned}
k^{*}(x) & =\underset{k}{\operatorname{argmax}}\left\{P\left(\mathbf{x} \in B_{k}\right)\right\} \\
& =\underset{k}{\operatorname{argmax}}\left\{p\left(B_{k} \mid f_{1}, \ldots, f_{W}\right)\right\} \\
& =\underset{k}{\operatorname{argmax}}\left\{\log p\left(f_{1}, \ldots, f_{W} \mid B_{k}\right)+\log p\left(B_{k}\right)\right\} \\
& =\underset{k}{\operatorname{argmax}}\left\{\sum_{i=1}^{W} \log p\left(f_{i} \mid B_{k}\right)+\log p\left(B_{k}\right)\right\}
\end{aligned}
$$

where $\mathbf{x}=\left(f_{1}, \ldots, f_{W}\right)$.

It is worth noting that the reasons we choose naive Bayes over other classification methods are: 1) implementation of a naive Bayes is rather easy and training can be done by calculating 


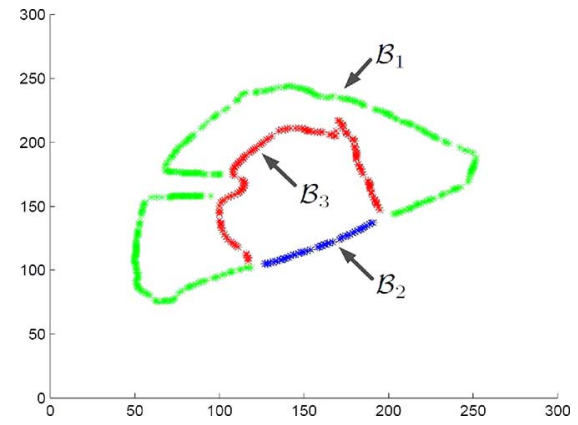

Fig. 2. All pixels are classified into four groups: $B_{1}, B_{2}, B_{3}, B_{4} . B_{1}$ represents the boundary points being between the weld pool region and the background, marked in green. $B_{2}$ represents the boundary points being between the arc light region and the background, marked in blue. $B_{3}$ represents the boundary points being between the weld pool region and the arc light region, marked in red. $B_{4}$ represents the non-boundary points and was not shown in this figure.

closed-form equations and 2) in a naive Bayes, features are assumed to be independent. The decoupling of the features allows us to further investigate how each selected feature contributes to the overall classification so that simplification or improvement of the classifier becomes possible.

Please notice that, for both of the schemes, we may lose a small amount of accuracy in the final probabilistic models. In the first scheme, errors are introduced when solving the over-constrained problem. In the second scheme, we reestablish a new classifier, instead of the strong classifier learned by AdaBoost. This may cause the loss of accuracy. This is acceptable because a "suboptimal" classifier often would be sufficiently good for driving an active contour.

\section{Weld Pool Visual Sensing Using Adaboost-Based ACTIVE CONTOURS}

This section presents the details of applying the proposed AdaBoost-based active contours to weld pool visual tracking in automatic arc welding.

\section{A. Boundary-Based Energy Term With Application-Dependent Prior Using Adaboost}

In a typical weld pool image, all pixels can be classified into four categories $B_{1}, B_{2}, B_{3}, B_{4}$ as follows.

- $B_{1}$ : the boundary between the weld pool region and the background, marked in green in Fig. 2.

- $B_{2}$ : the boundary between the arc light region and the background, marked in blue in Fig. 2.

- $B_{3}$ : the boundary between the weld pool region and the arc light region, marked in red in Fig. 2.

- $B_{4}$ : the nonboundary points.

The boundary points $B_{1} \cup B_{2} \cup B_{3}$ are defined as all of the edge points detected by Canny edge detectors and their immediate neighbors. In practice, this can be done using Canny edge detection followed by a morphological dilation. In doing so, we can benefit from the advantages of conventional local edge detectors[15].

The desired boundary that we refer to as weld pool boundary is composed of two classes $B_{1}$ and $B_{2}$. This boundary preference information is regarded as what we called application-dependent prior. Following the AdaBoost approach described in

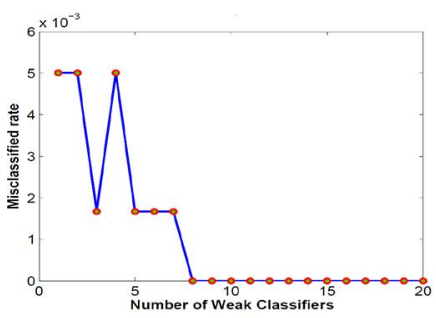

(a)

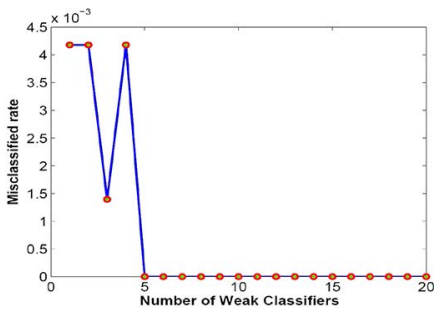

(b)

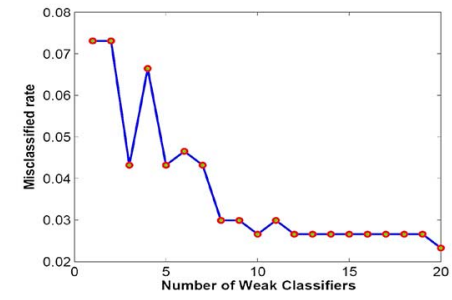

(c)

Fig. 3. Performance of the strong classifiers against the number of the weak classifiers that were used to create the strong classifier. The $x$-axis represents the number of the weak classifiers. The $y$-axis represents the misclassification rate. (a) $B_{1}$ versus $B_{2}$. (b) $B_{1}$ versus $B_{3}$. (c) $B_{2}$ versus $B_{3}$.

Section III, we can build a probabilistic model for each class based upon a few well-selected features.

In order to manipulate the pixel information to build the statistical model, the pixels should be characterized in some way. In our application, we propose to use the following features to describe each pixel, i.e.,

$$
\begin{aligned}
F(\mathbf{x}) & =\left(f_{1}, f_{2}, \ldots, f_{M+4}\right) \\
& =\left(x, y, I(\mathbf{x}),\|\nabla(I(\mathbf{x}))\|, f_{1}^{H}, \ldots, f_{M}^{H}\right)
\end{aligned}
$$

where $x$ and $y$ denote the position of the pixel, $I(\mathbf{x})$ denotes the intensity, $\|\nabla(I(\mathbf{x}))\|$ denotes the magnitude of the gradient at the pixel, and $f_{i}^{H}, i=1,2, \ldots, M$ denote Haralick features calculated at $\mathbf{x}$; see [26].

A series of frames are first manually segmented and labeled. Then, following the AdaBoost approach described in Section III, Gaussian Bayesian classifiers are trained to separate each pair of the classes. Fig. 3 shows the misclassification rate against the numbers of the weak classifiers. More classifiers, hence more features selected generally increases the accuracy of classification. On the other hand, a balance must be reached between the accuracy and the amount of features used. It is preferable to use fewer features as long as certain accuracy can be reached. By analyzing the results of AdaBoost, it is found that even as few as two features can reach sufficiently accurate classification in our application. These two crucial features are intensity and the Haralick feature Contrast. Fig. 4 shows these two features on one typical frame. One feature is intensity which separates the class $B_{1}$ and $B_{2}$, as well as $B_{1}$ and $B_{3}$. Another one is called Contrast which is crucial for separating the class $B_{2}$ from $B_{3}$. These results generated by AdaBoost agree with our experimental observations very well. Fig. 5 shows graphically how boundary pixels distributed in the space spanned by these two features. It can be observed that it is possible to distinguish the pixels belonging to different boundary classes. In fact, based upon these two crucial features, 


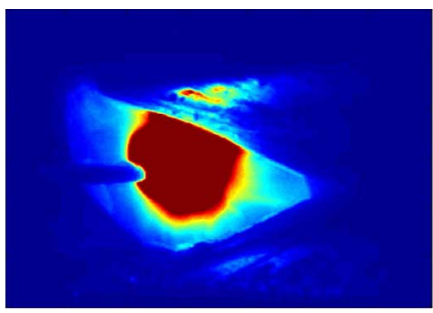

(a)

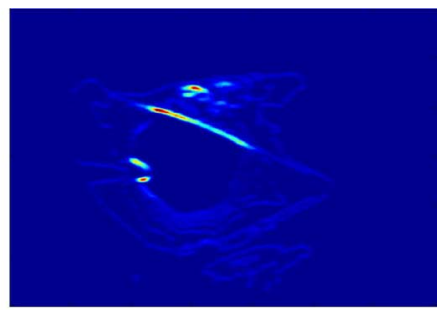

(b)

Fig. 4. Crucial features selected by AdaBoost. (a) The intensity which is crucial to separate the classes $B_{1}$ and $B_{2}$, as well as $B_{1}$ and $B_{3}$. (b) The second Haralick feature Contrast which is crucial to separate the classes $B_{2}$ and $B_{3}$.

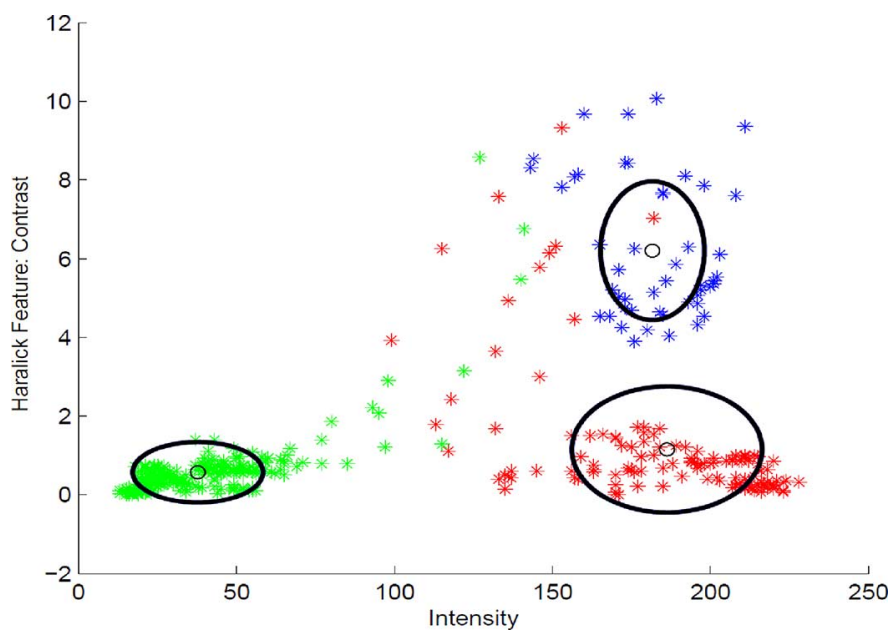

Fig. 5. Boundary pixels in different classes described by the intensity and the second Haralick feature contrast. The black ellipses indicate the 2-D Gaussian distribution of each class learned from labeled data. Note that, due to the assumption that different features are independent in naive Bayes classifiers, the covariance matrices here are diagonal matrices.

a naive Bayes classifier can be constructed. The probabilistic models are given as

$$
\left\{u_{k}(\mathbf{x})=P\left(\mathbf{x} \in B_{k}\right), k=1,2,3\right\} .
$$

The probability that a pixel $\mathbf{x}$ is situated on the true desired boundary is then obtained by

$$
p_{\text {app }}(\mathbf{x})= \begin{cases}\Psi\left(u_{1}(\mathbf{x}), u_{2}(\mathbf{x})\right), & \text { if } \mathbf{x} \in B_{1} \bigcup B_{2}, \\ 0, & \text { if } \mathbf{x} \in B_{3} \bigcup B_{4}\end{cases}
$$

where $\Psi$ stands for the methods for constructing a probability such as $\max (\cdot)$. The key idea is that we penalize the pixels in the classes $B_{3}$ and $B_{4}$ much heavier than the pixels in the classes $B_{1}$ and $B_{2}$. Then, this penalization can be related to a probability distribution such as Gibbs distribution as in Markov random field [5]. Inspired by the work on geodesic active regions [43], we define the boundary-based energy functional as follows:

$$
E_{\text {boundary }}=\int_{0}^{1} g\left(p_{\text {app }}(\mathbf{x}(s))\right)|\dot{\mathbf{x}}(s)| d s
$$

where $g(\cdot)$ is a monotonically decreasing function, for example,

$$
g(x)=\frac{1}{1+x^{p}} \quad p=1 \text { or } 2 .
$$

\section{B. Region-Based Energy Term}

Using the boundary-based force defined above, the active contour will be attracted by the correct boundaries. However, the capture range of this force is small, i.e., the contour has to be close enough to the boundaries to make the force take effect. This makes the initialization very critical but difficult, especially in a fully automated system such as in our application. Therefore, enlarging the capture range of the active contour is important. The capture range is also related to the convexity of the energy functional discussed in Section II. Roughly speaking, the capture range is the subregion of the entire image where the energy functional is convex. If the energy functional is designed properly, i.e., being convex in a large subregion of the image, the initialization will become much easier. Existing techniques for enlarging the capture range include adding an extra inflating force [15], gradient vector flow [58] and region-based techniques [13], [43], [62]. In our application, we employ region-based techniques to both enlarge the capture range and enhance the ability to capture boundaries.

Suppose that we are dealing with binary segmentation problem. The entire image is separated into two regions, denoted as $\Omega_{\text {in }}$ and $\Omega_{\text {out }}$, and the boundary $\mathcal{C}$. In each of the regions $\Omega_{\text {in }}$ and $\Omega_{\text {out }}$, we further assume that the intensity $I(\mathbf{x})$ at each pixel, considered as a random variable, is independently and identically distributed, denoted as $p_{\text {in }}$ and $p_{\text {out }}$, respectively. The likelihood of the whole image given the segmentation $\mathcal{C}$ is computed by

$$
\begin{aligned}
P(I \mid \mathcal{C}) & =\prod_{\mathbf{x} \in \Omega} p(I(\mathbf{x}) \mid \mathcal{C}) \\
& =\prod_{\mathbf{x} \in \Omega_{\text {in }}} p_{\text {in }}(I(\mathbf{x})) * \prod_{\mathbf{x} \in \Omega_{\text {out }}} p_{\text {out }}(I(\mathbf{x})) .
\end{aligned}
$$

To maximize this likelihood is equivalent to minimizing its negative logarithm. Then, we have

$$
\begin{aligned}
E_{\text {region }} & =-\int_{\Omega_{\text {in }}} \log p_{\text {in }}(I(\mathbf{x})) d \mathbf{x}-\int_{\Omega_{\text {out }}} \log p_{\text {out }}(I(\mathbf{x})) d \mathbf{x} \\
& =-\int_{\Omega_{\text {in }}} \log p_{\text {in }}(I(\mathbf{x})) d \mathbf{x}+\int_{\Omega_{\text {in }}} \log p_{\text {out }}(I(\mathbf{x})) d \mathbf{x} \\
& =-\int_{\Omega_{\text {in }}} \log \frac{p_{\text {in }}(I(\mathbf{x}))}{p_{\text {out }}(I(\mathbf{x}))} d \mathbf{x} .
\end{aligned}
$$

Note that the constant term has been eliminated from the above equations since it does not affect the minima of the energy functional.

The problem now is to model and estimate the intensity distributions over different regions $p_{\text {in }}(I(\mathbf{x}))$ and $p_{\text {out }}(I(\mathbf{x}))$.

1) Modeling Weld Pool Images Statistically: To investigate the histograms of different regions, we manually segment the weld pool images into the foreground and the background and compute the histograms respectively.

The Euclidean distances between two histograms are computed and presented as a distance matrix, see Fig. 6 . The first 15 rows or columns are foreground histograms, the rest are background histograms. The similarity within one group and the dissimilarity between two groups can be easily observed in this distance matrix. This observation shows that the overlap between the histograms of the foregrounds and the backgrounds is small. 


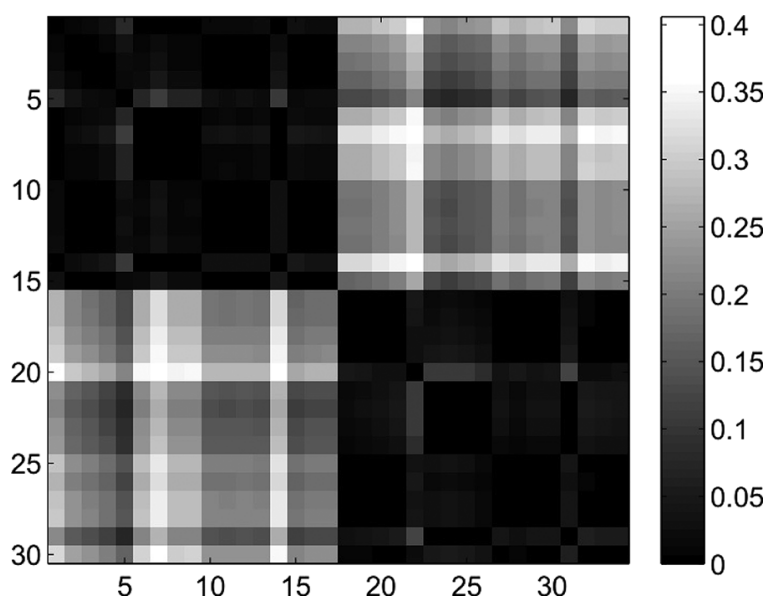

Fig. 6. Euclidean distances between each two histograms are computed and presented as a distance matrix. The first 17 rows or columns are foreground histograms, the rest are background histograms. The similarity within one group and the dissimilarity between the groups can be easily observed in this distance matrix.

It is actually possible to use the histogram as the model of each region to drive active contours, using the approach proposed in [11]. In addition, considering that there are actually two different light sources, the arc and the weld pool, existing in our application, we propose to employ Gaussian mixture models to model the intensity distributions of the foreground and background regions.

The Gaussian mixture models are commonly used parametric modeling tools for density estimation [5]. The Gaussian mixtures are constructed as a linear superposition of Gaussian components in the form

$$
p(\mathbf{x})=\sum_{i=1}^{N} \pi_{i} \mathcal{N}\left(\mathbf{x} \mid \mu_{i}, \Sigma_{i}\right)
$$

where $N$ is the number of the Gaussian components. $\pi_{i}, i=$ $1, \ldots, N$ are the mixing coefficients which satisfies that

$$
0 \leq \pi_{i} \leq 1, \quad \sum_{i=1}^{N} \pi_{i}=1
$$

Typically, the parameters of the models are estimated in terms of maximum likelihood using Expectation Maximization (EM) [5].

In our application, the number of the Gaussian components was selected as 2 because of the two kinds of light sources, namely the arc and the weld pool. This leads to the mixture of Gaussian model in the form

$$
I(x, y) \sim \pi_{1} \mathcal{N}\left(\mu_{1}, \Sigma_{1}\right)+\pi_{2} \mathcal{N}\left(\mu_{2}, \Sigma_{2}\right) .
$$

Both the foreground and background regions are modeled as Gaussian mixture models. The trained models are presented in Fig. 7 where the two components of the Gaussian mixture models of the weld pool region are shown. One component situated its center as 81.5 which corresponds to the molten pool light source, the other one 254.45 corresponding to the arc light

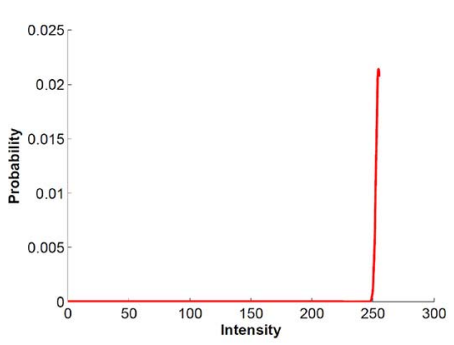

(a)

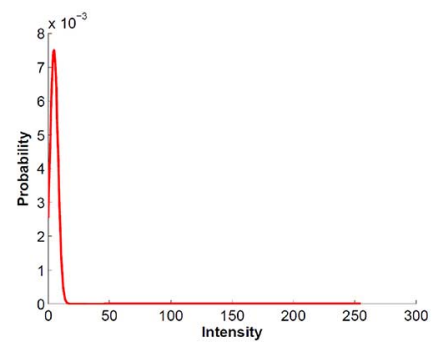

(c)

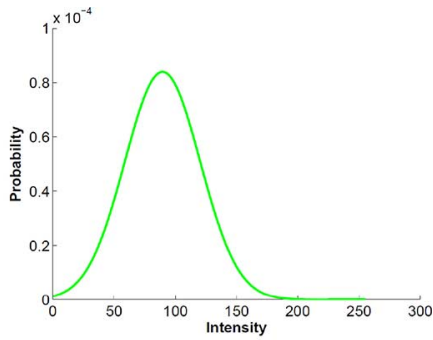

(b)

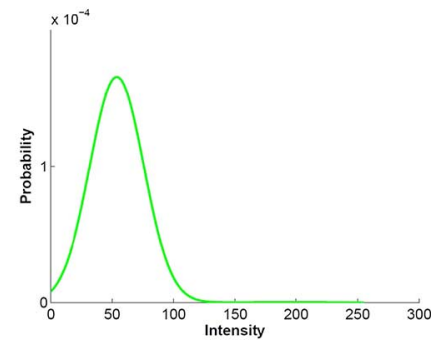

(d)
Fig. 7. Modeling the weld pool region and the background using Gaussian mixture models which has two isotropic Gaussian components. (a), (b) Weld pool: $0.3054 * \mathcal{N}\left(254.45,7.9^{2}\right)+0.6946 * \mathcal{N}\left(81.5,46.7^{2}\right)$. (c), (d) Background: $0.8738 * \mathcal{N}\left(4.79,4.6^{2}\right)+0.1262 * \mathcal{N}\left(53.9,31^{2}\right)$. (a) Weld pool: component 1. (b) Weld pool: component 2. (c) Background: component 1. (d) Background: component 2 .

source. This is consistent with our observation. The mixing coefficient 0.6961 reflects the proportion between the area of the molten pool and the arc light.

\section{Unifying Region and Boundary-Based Information}

The total energy incorporating both the boundary and the region based information is then defined by

$$
\begin{aligned}
E & =\eta E_{\text {boundary }}+\gamma E_{\text {region }} \\
& =\eta \int_{0}^{1} g\left(p_{b}(\mathbf{x})\right)|\dot{\mathbf{x}}(s)| d s-\gamma \int_{\Omega_{\text {in }}} \log \frac{p_{\text {in }}(I(\mathbf{x}))}{p_{\text {out }}(I(\mathbf{x}))} d \mathbf{x} .
\end{aligned}
$$

The first term is the boundary-based force where the boundary classification information is incorporated. The second term is the region-based force where the region classification information is incorporated. To balance the contributions of these two terms to the curve evolution, we introduce the weights, $\eta$ and $\gamma$.

The reason that parametric representation, instead of geometric representation of active contours is chosen for this application is two fold. First, the most significant advantage of geometric representation or level set method is that it can handle topological changes conveniently. However, because topological changes do not exist in this application, and also because typically parametric implementation is much faster than level set methods, it is preferable to use parametric representation here. Minimizing the above energy functional by gradient descent leads to the following Euler-Lagrange equation:

$$
\frac{\partial \mathbf{x}(t)}{\partial t}=\eta \underbrace{\left\{g\left(p_{b}(\mathbf{x})\right) \kappa-\nabla g\left(p_{b}(\mathbf{x})\right) \cdot \overrightarrow{\mathbf{n}}\right\} \overrightarrow{\mathbf{n}}-\gamma \underbrace{\log \frac{p_{\text {in }}(I(\mathbf{x}))}{p_{\text {out }}(I(\mathbf{x}))} \overrightarrow{\mathbf{n}}}_{\text {region-based force }}}_{\text {boundary-basedforce }}
$$


where $\kappa$ denotes the curvature of the curve at the point $\mathbf{x} \cdot \overrightarrow{\mathbf{n}}$ denotes the unit normal vector.

By expanding (21), we obtain the following equation:

$$
\begin{aligned}
\frac{\partial \mathbf{x}(t)}{\partial t}=\eta_{1} \underbrace{g\left(p_{b}(\mathbf{x})\right) \kappa \overrightarrow{\mathbf{n}}}_{\text {geodesic internal force }}-\eta_{2} \underbrace{\left(\nabla g\left(p_{b}(\mathbf{x})\right) \cdot \overrightarrow{\mathbf{n}}\right) \overrightarrow{\mathbf{n}}}_{\text {boundary-based force }} \\
-\gamma \underbrace{\log \frac{p_{\text {in }}(I(\mathbf{x}))}{p_{\text {out }}(I(\mathbf{x}))} \overrightarrow{\mathbf{n}}}_{\text {region-based force }} .
\end{aligned}
$$

Note that the first term involves both the curvature $\kappa$ of the curve and the boundary-based potential $g\left(p_{b}(\mathbf{x})\right)$. Roughly speaking, it serves as an internal force in a Riemannian manifold and is therefore referred to as geodesic internal force. The second term is nothing but the project of the boundary-based force vector onto the normal direction. The third term is the region-based force. Note that the weight $\eta$ is replaced by two weights $\eta_{1}$ and $\eta_{2}$ to further balance the first two terms. It can be seen that this is consistent with the general framework of active contours as shown in (1). In practice, the weights were carefully determined by trial and error. If necessary, we can replace the geodesic internal force with the Euclidean internal force as shown in (1). In numerical implementation, the (22) is discretized using the finite difference method and solved iteratively [30], [34].

\section{EXPERIMENTAL RESULTS}

Here, we shall discuss the experimental works for validating the proposed approach. In addition to implementing the evolving equation in (22), we made two small modifications as follows.

1) After a number of iterations using both region-based and boundary-based information, we remove the region-based information and allow the boundary-based information to take effect only. Doing this can help better capture the small details of the weld pool boundary.

2) One more force called anchor force is added to facilitate active contours to form the $\mathbf{U}$ shape where the wire is located. The idea is that in our application the camera is fixed relative to the welding torch. The position of the torch tip in the image is therefore fixed. We put an extra constraint that if the control points of the active contour are close enough to the anchor, they will be captured by the anchor and stop moving.

It is noted that the welding wire boundary is also important for automating the arc welding process and therefore extracted by the algorithm as well. But when the temporal-spatial errors are computed, as will be defined in Section V-C, the welding wire boundary is excluded because the boundary of the wire is not of essential interest in identifying the boundary of the weld pool.

\section{A. Experiment Setup}

Fig. 8 shows the experiment platform which consists of a CCD camera the speed of which is up to 90 fps equipped with an optical filter, a manipulator and a welding machine. The optical filter was carefully selected by analyzing the spectrum of the lights emitting during welding. With the help of the optical filter

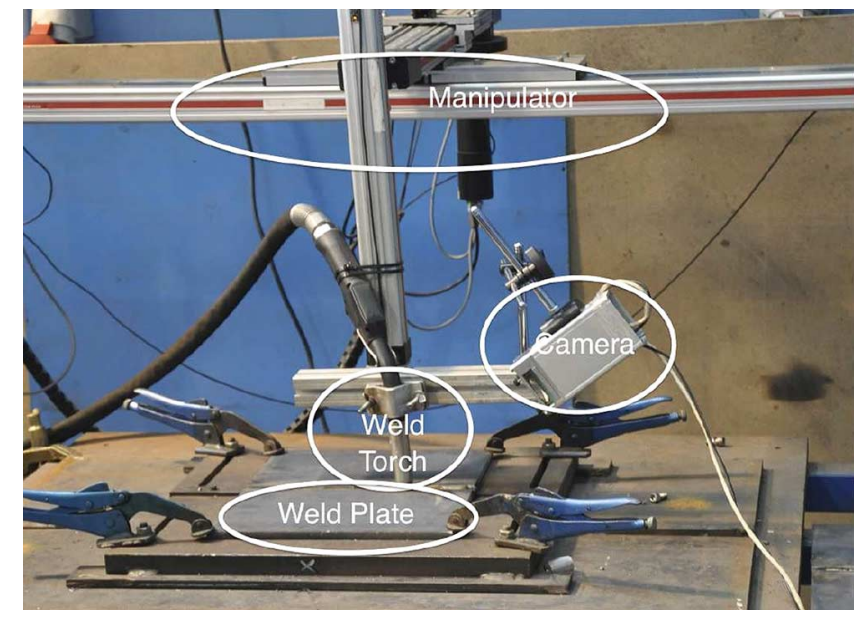

Fig. 8. Configuration of the experiment platform, including an industrial CCD camera equipped with an optical filter, a manipulator, and a welding machine.

and online control of the camera parameters, the interference of the arc light was partially suppressed and relatively good weld pool images were captured. The captured raw images are 8-bit grayscale images of size around $700 \times 500$ pixels. A pre-processing program developed for the whole system including both seam tracking and weld pool sensing is responsible for cropping out the weld pool region which is typically $200 \times 200$ pixels.

\section{B. Evaluation Criteria}

As the weld pool shape may change a lot from frame to frame and in different experiments, an uniform ground truth does not exist. The evaluation of the accuracy of the proposed algorithms therefore mostly relies on the welding expert inspection. On the other hand, to quantitatively measure the performance of a weld pool boundary tracking algorithm, a reference boundary for each frame is manually marked by segmenting the weld pool boundary based on welding experts inspection. This serves as a quantitative measurement of the accuracy of an algorithm based on welding expert inspection. The detailed discussions of marking and comparing weld pool boundaries are presented in Section V-C.

It is worthwhile to note that even though we still refer to a reference boundary as a ground truth, it is in fact a quantitative representation of welding experts inspection, not the true weld pool boundary. The boundary extracted by algorithms can be more accurate than the one extracted by welding experts especially when the boundary is visually weak. In addition, it is important to point out that the weld pool boundary can be vague in some frames and the transition region may span up to more than ten pixels, see Fig. 9. Therefore, as long as the error in pixel is less than, say ten pixels, we still think that the weld pool tracking algorithm agrees well with the human experts inspection.

Two different experiments have been conducted and used for the evaluation of the proposed weld pool tracking algorithm based on AdaBoost-based active contour. The important details of these two experiments are listed here.

1) Experiment 1. The first experiment was made as a root path short-circuit GMAW on a narrow v-groove seam. The torch approximately followed the center line of the seam 


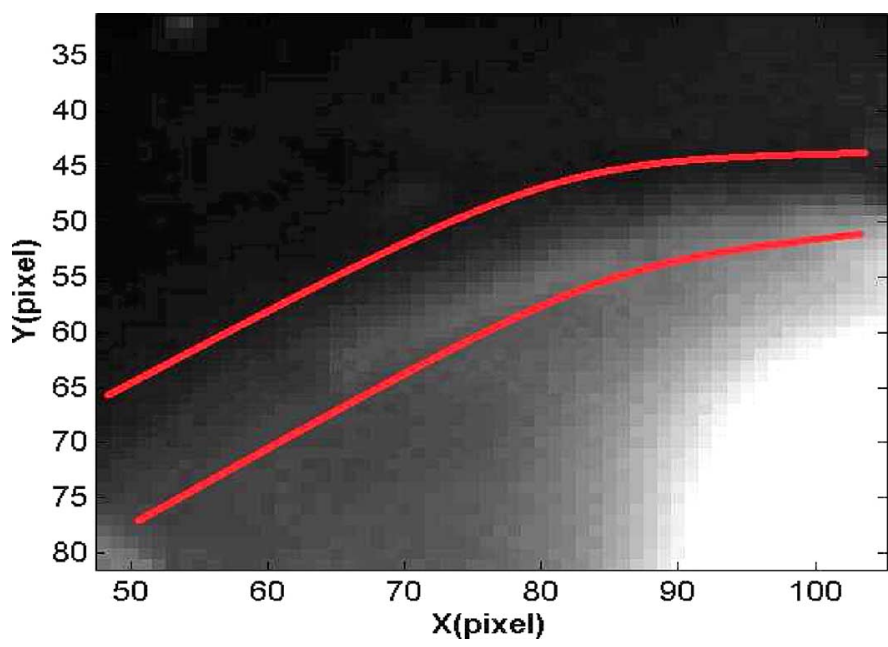

Fig. 9. Segment of a weld pool boundary. Between the two red curves are the transition region of the edge. It can be seen that the edge is not very sharp and can span up to 10 pixels.

without oscillation. The characteristics of the weld pool images are as follows. First, the weld pool is almost symmetrical and slowly changed from frame to frame. Second, strong arc light exists above the weld pool region.

2) Experiment 2. The second experiment was again made as a root path short-circuit GMAW on a narrow v-groove seam, but in this case the path of the torch was a little away from the center line and biased to one side. The consequence is that the arc is generated and the weld pool is deposited on one side of the seam. Appearing on the images, the weld pool is asymmetrical.

In both experiments, the same parameters have been used. The wire-feed speed is $5.5 \mathrm{~m} / \mathrm{min}$. The wire diameter is 1.0 $\mathrm{mm}$. The welding voltage is $17.5 \mathrm{~V}$. The shielding gas is $\operatorname{Argon}(82 \%) / C O 2(18 \%)$. Here we shall make a very brief introduction to the Gas Metal Arc Welding process. During short-circuit GMAW, the metal wire tip touches the weld pool or the plate to form a short circuit which then causes very strong arc light and a large amount of heat. The tip would then be melted down and drop into the weld pool. The arc light would then vanish. This process is repeated dozens of times per second. Due to this periodic characteristic, we could do the quantitative comparison on the frames which cover a few periods and capture variations of the weld pool shape and arc light.

\section{Setting the Ground Truth: Manual Segmentation of Weld Pools}

To quantitatively measure the performance of the proposed approach compared to welding experts inspection, we shall manually segment the weld pool boundaries. Here, we discuss this quantitative measurement, i.e., how to compare a weld pool extracted by the proposed approach with the corresponding ground truth, the manually segmented shape.

In essence, the evaluation is based on comparison between shapes. A common technique for shape comparison is so-called Procrustes analysis [31]. In general, shape comparison relies on two key elements. First, a certain adjustment, so called shape alignment, must be performed to eliminate the shape difference caused by interfering factors such as the placement in space and the size of the shapes. Second, a certain metric must be defined properly to capture the shape difference, such as Procrustes distance. Mathematically, shape comparison can be expressed as follows:

$$
\left\|\mathbf{X}-\mathbf{X}_{\mathrm{G}}\right\|_{\psi}=\psi\left(\Gamma(\mathbf{X}), \Gamma\left(\mathbf{X}_{\mathrm{G}}\right)\right)
$$

where $\mathbf{X}_{\mathrm{G}}$ stands for the manually segmented shape. $\psi(\cdot)$ or $\|\cdot\|_{\psi}$ denotes a certain metric. $\Gamma$ denotes all allowed transformation of the shapes such as translation, rotation, reflection and uniform scaling.

It is worth pointing out that the metric $\psi$ can be selected very broadly. If we consider a curve as a function, any functional that satisfies the conditions required for being a distance is possible. However, if we, as in many shape analysis literatures, think of a shape as a set of special points, so called landmarks, it is natural to measure the shape difference on all the landmarks. The landmarks are special in the sense that they can characterize a shape somehow. For instance, if we describe the shape of a desk, possible landmarks are the corners. By linking all these corners, we will have a sketch of a desk. All of the statistical measurement can be performed on these landmarks. Using landmarks is very common in shape analysis of rigid objects [49].

However, the weld pool in our application is nonrigid fluid metal. It is difficult to describe a weld pool shape by regular landmark based methods, even though the weld pool boundary is indeed represented by a set of discrete points, i.e., a firstorder spline. An alternative discussion on spline-based curve comparison can also be found in [6].

In this work, a measurement based on representing all of the points in a polar coordinate system $(r, \theta)$, as shown in Fig. 10, is proposed. The pole is selected at the tip of the welding wire. It is convenient because the position of the welding wire tip in the image plane keeps unchanged due to the fact the camera is mounted to the welding torch. The polar axis is determined as along the welding wire, see the red ray shown in Fig. 10. The resolution of $\theta$ is determined as $7.2^{\circ}$, i.e., 50 rays in our application. All the control points extracted by an automatic algorithm or manually segmentation will be projected onto the closest ray. We can measure the difference of the shapes by measuring difference of the corresponding radial coordinates, i.e., $\left(\Delta r_{1}, \Delta r_{2}, \ldots, \Delta r_{N}\right)$ where $N$ is the amount of rays. This permits us to generate statistics such as mean and variance to compare two shapes quantitatively. For the evaluation of an algorithm, the accuracy or error can be defined as

$$
\text { Error }_{\text {temporal-spatial }} \equiv\left[\begin{array}{cccc}
\Delta r_{11} & \Delta r_{12} & \ldots & \Delta r_{1 N} \\
\Delta r_{21} & \Delta r_{22} & \ldots & \Delta r_{2 N} \\
\vdots & \vdots & \ddots & \vdots \\
\Delta r_{T 1} & \Delta r_{T 2} & \ldots & \Delta r_{T N}
\end{array}\right]
$$

where $T$ denotes the number of frames, and $N$ denotes the number of control points. A box plot computed in each row indicates how errors are distributed in each frame, i.e., the spatial error distribution. A box plot computed in each column indicates how errors are distributed at each control point, i.e., the 


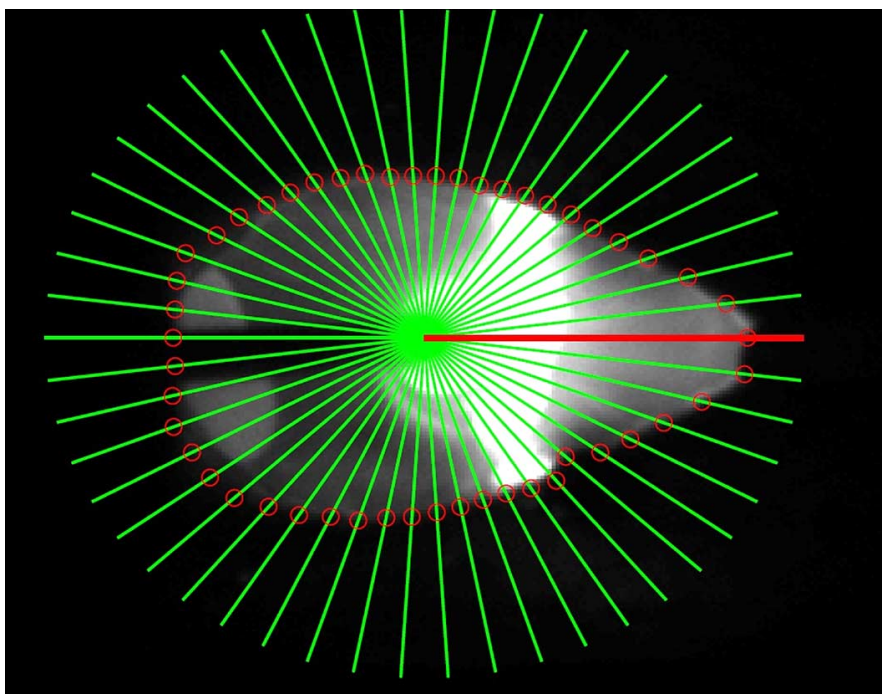

Fig. 10. Example of manually segmenting a weld pool boundary and representing all the points in a polar coordinate system $(r, \theta)$. The resolution of the polar angle $\theta$ is $7.2^{\circ}$, i.e., 50 rays. The polar axis is indicated in a red line segment. The rays are marked in green. The landmark-like points which are extracted manually and describe the weld pool boundary are marked in red circles.

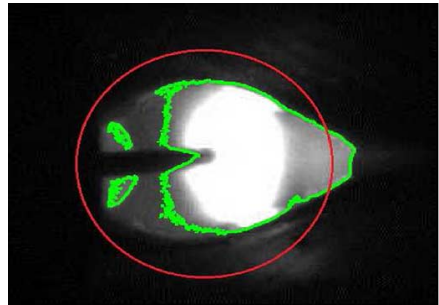

(a)

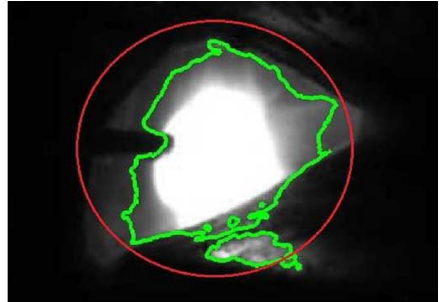

(b)
Fig. 11. Segmentation result of using the Chan-Vese model. The initial contour is marked in red circle. The final result is marked in green curves. It can be seen that the $\mathrm{CV}$ model failed to extract the weld pool boundary.

temporal error distribution. The spatial-temporal error distributions are used in evaluating a welding pool boundary tracking approach in this article.

Fig. 10 gives an example of manually segmenting a weld pool boundary and representing all the points in a polar coordinate system. The polar axis is indicated in a red line segment. The rays are marked in green. The landmark-like points are marked in red circles.

\section{Results}

Before presenting and discussing the results of the proposed algorithm, as an intuitive comparison, we show the result of segmenting a weld pool image using the Chan-Vese model which can detect object boundaries not necessarily defined by gradient [13].

Fig. 11 shows the result. The initial curve is marked in red. The final contour is indicated in green. Clearly the Chan-Vese model missed some parts of the desired boundary and were attracted by some undesired edges. This is due to the fact that the Chan-Vese model attempts to find a boundary between two homogeneous regions. In our application the region enclosed by a weld pool boundary is clearly not homogeneous.

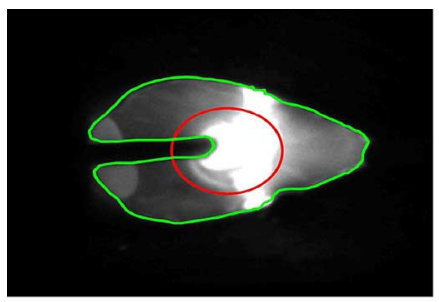

(a)

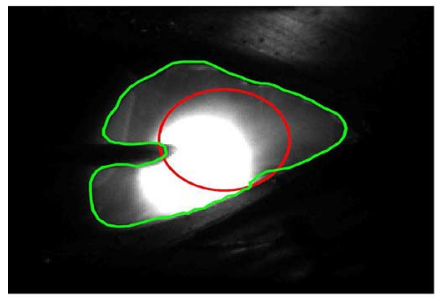

(c)

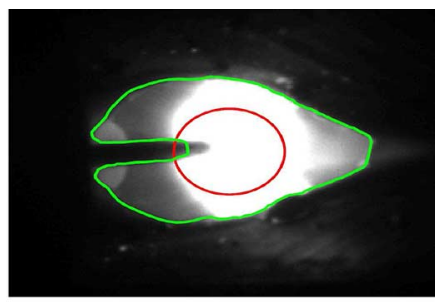

(b)

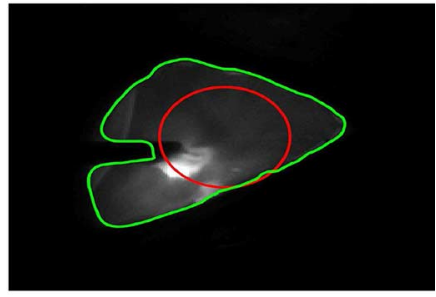

(d)
Fig. 12. Results of the proposed approach on a few typical frames. The initial contours are indicated in the red circles. The final contours are marked in green curves. $(a-b)$ Results in the experiment 1. $(c-d)$ Results in the experiment 2. (a) Experiment 1, Frame 66. (b) Experiment 1, Frame 67. (c) Experiment 2, Frame 55. (d) Experiment 2, Frame 56.

Recall that thanks to the periodic characteristic of short-circuit GMAW, quantitative comparison has been done on the frames which were captured in a few periods of short-circuit arc welding. We manually segmented a series of frames in the experiment 1 and 2 and then compared the weld pool boundaries extracted by the proposed approach to the manually segmented boundaries. The results of testing the proposed approach are presented in Figs. 12-14.

Fig. 12 shows a few tracking results from both experiments. The initial contours are marked in red circles and the final contours are marked in green curves. It can be seen that the final results agrees the weld pool boundary very well. In Figs. 13 and 14 , we also plotted boxplots along each, temporal or spatial, dimension. It can be observed that the medians of both the temporal and spatial errors are around $1 \sim 2$ pixels with a few exceptions which are less than 10 pixels. As we discussed previously, due to the vagueness of the weld pool boundary in some cases, these exceptions are reasonable and acceptable. We can conclude that the proposed algorithm agrees the welding expert inspection very well in both experiment 1 and 2 .

Finally, we present a figure where all of the extracted weld pools are plotted in the image domain in a sequence with a predefined simulated shift in the $X$ - xis in Fig. 15 to serve as an intuitive illustration. The similarity between the resulting figure and the real weld beads can be observed. More tracking results can be found in Fig. 16.

\section{E. Run-Time Analysis}

The proposed approach consists of two stages: the off-line training and the on-line running. The time complexity of the two phases are $\mathrm{O}\left(T_{h} n_{f} n_{c}^{2}\right)$ and $\mathrm{O}\left(n_{t} n_{p}\right)$ respectively, where $T_{h}$ is the number of hypotheses, $n_{f}$ is the number of features, $n_{c}$ is the number of classes, $n_{t}$ is the number of the active contour iteration, and $n_{p}$ is the number of the active contour control points. 


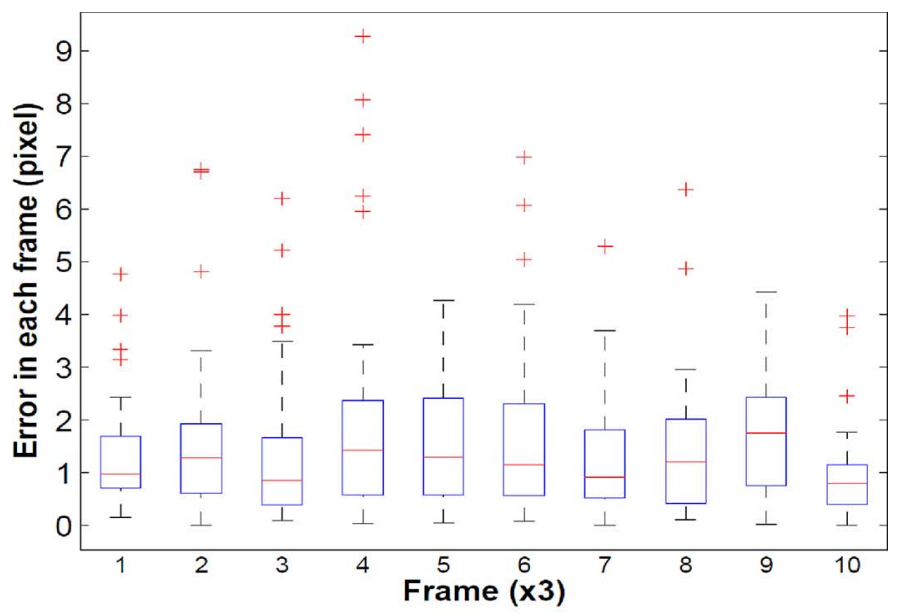

(a)

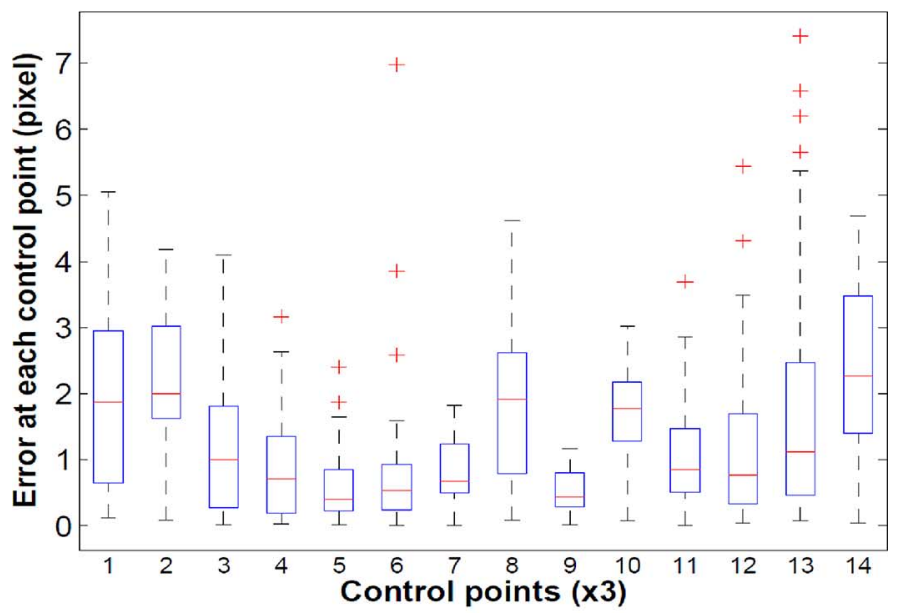

(b)

Fig. 13. Results in the experiment 1. (a) Error distribution at each frame. (b) Error distribution at each control point along the boundary.

In practice, we implemented both phases in MATLAB except for computing the Haralick feature which is implemented in $\mathrm{C}++$. In off-line training, two crucial features which convey most discriminant information were selected and a naive Bayes classifier based on these two features were built. Computing the Haralick feature contrast on a $200 \times 200$ weld pool image costs around $7 \mathrm{~ms}$ on a regular desktop PC which has Intel i7 CPU and $4 \mathrm{G}$ RAM. It is worth noting that the usual way of computing Haralick features is to compute gray-level co-occurrence matrix first, but this is extremely inefficient in our application for two reasons.: 1) we are interested in contrast only and 2) when computing a gray-level co-occurrence matrix around a pixel, only a few neighboring pixels are involved and the resulting co-occurrence matrix is therefore extremely sparse. Therefore, the Haralick feature is calculated directly according to its equation in our application. The feature-selective AdaBoost took less than one second. Since the off-line training needs to be done only once, the total processing time is not a major concern.

For on-line running, the active contour cost around one second for a typical weld pool frame in non-optimized MATLAB code. For real time applications, if implemented efficiently in $\mathrm{C}$ or $\mathrm{C}++$, real-time performance is achievable.

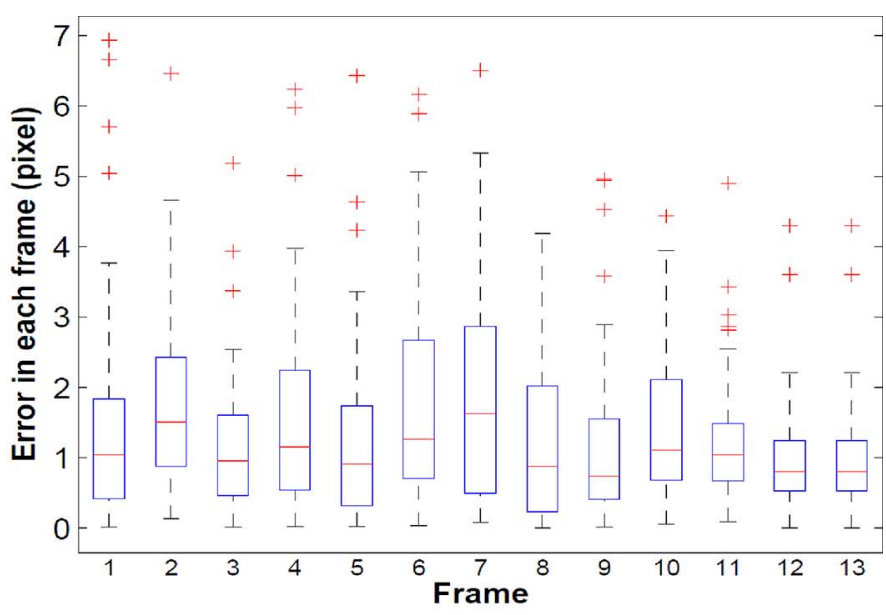

(a)

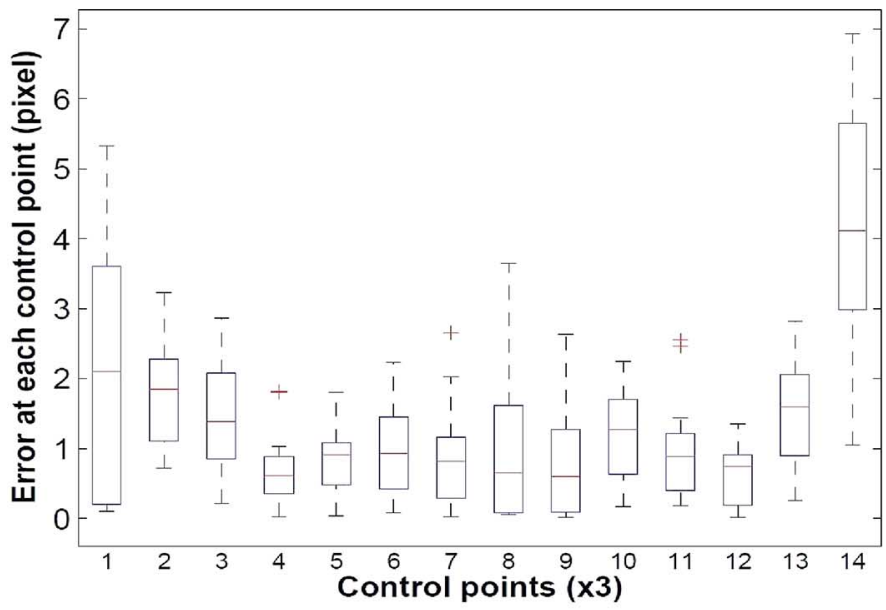

(b)

Fig. 14. Results in the experiment 2. (a) The error distribution at each frame. (b) The error distribution at each control point along the boundary.

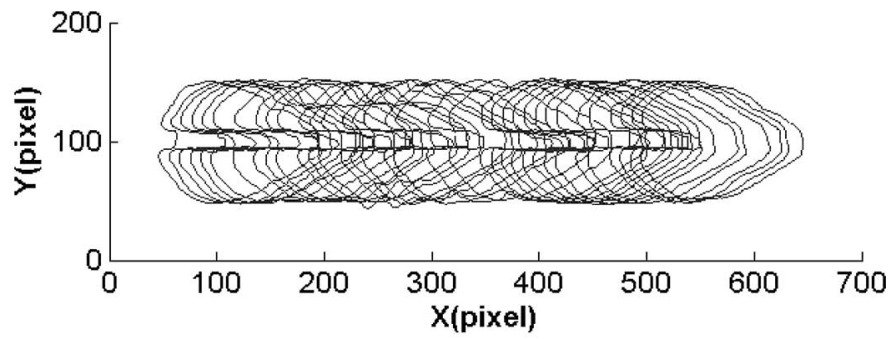

(a)

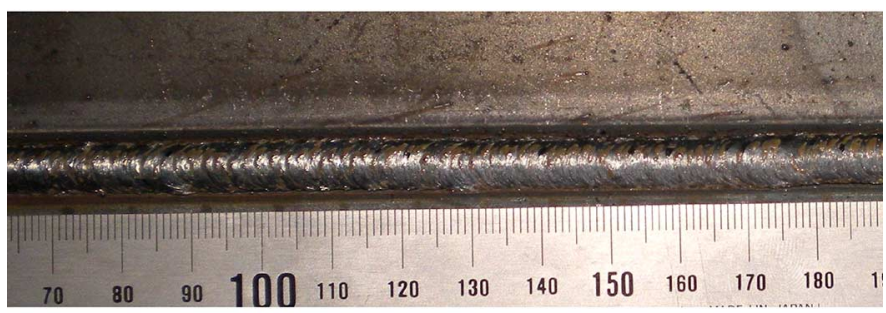

(b)

Fig. 15. (a) Series of the extracted weld pools are plotted in the image domain in a sequence with a predefined simulated shift in the $X$-axis. The similarity between the resulting figure and the real weld bead can be observed. (b) Typical weld bead. 


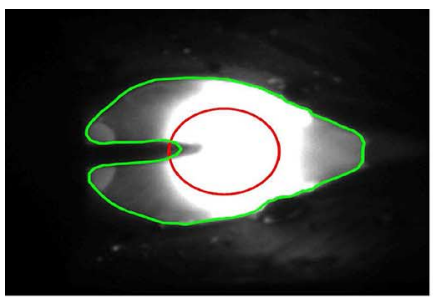

(a)

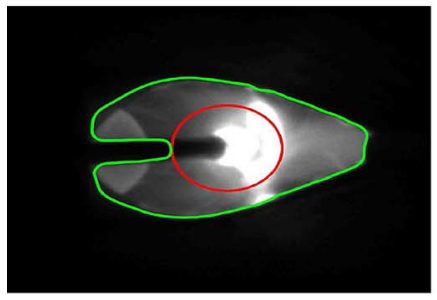

(c)

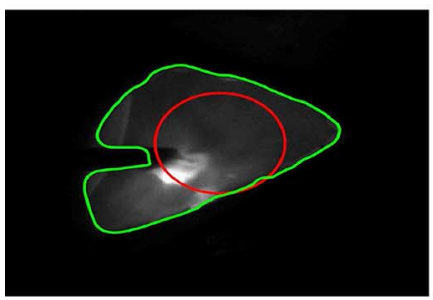

(e)

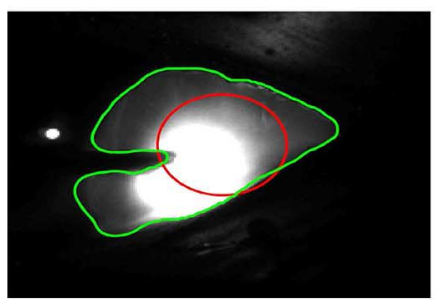

(g)

Fig. 16. Ttracking results of the proposed approach. The initial contours are indicated in the red circles. The final contours are marked in green curves. $(\mathrm{a}-\mathrm{d})$ Results in the experiment 1. $(\mathrm{e}-\mathrm{h})$ Results in the experiment 2. (a) Experiment 1, Frame 68. (b) Experiment 1, Frame 69. (c) Experiment 1, Frame 127. (d) Experiment 1, Frame 128. (e) Experiment 2, Frame 56. (f) Experiment 2, Frame 58. (g) Experiment 2, Frame 59. (h) Experiment 2, Frame 60.

\section{CONCLUSION}

In this paper, we have proposed a solution to the weld pool visual tracking problem. An active contour has been extended with an application-dependent prior term. We regard the prior as a classification problem and model this using feature selective Adaboost. This approach boosts the speed of the tracking approach and facilitates a real-world application. The reported experiments verify the proposed method. The proposed method also gives suggestions to other applications on the importance of integrating application-dependent prior to reject disturbances or noises, and provides an examplar way of representing the prior as an AdaBoost classifier. Other applications can even involve examples in medical image processing such as boundary extraction of echocardiographic images, beyond industrial applications of 2-D and 3-D weld pool sensing, etc. For further works, more experiments will be needed to estimate the perfor- mance in real world welding applications. Moreover, we will investigate the possibility of adaptively determining the parameters of the proposed active contour using heuristics such as evolutionary algorithms.

\section{REFERENCES}

[1] B. Abdullah, J. Smith, W. Lucas, J. Lucas, and M. Houghton, "A lowcost vision system for real-time monitoring of welding applications," in Proc. 14th Int. Conf. Joining of Mater. and 5th Int. Conf. on Education in Welding, Helsingor, Denmark, Apr. 2007, p. 13.

[2] B. Abdullah, J. Smith, W. Lucas, J. Lucas, and F. Malek, "Monitoring of TIG welding using laser and diode illumination sources: A comparison study," in Proc. Int. Conf. Electron. Design, Dec. 2008, pp. 1-4.

[3] M. S. Allili and D. Ziou, "Object tracking in videos using adaptive mixture models and active contours," Neurocomput., vol. 71, no. 10-12, pp. 2001-2011, Jun. 2008.

[4] E. Bae and X.-C. Tai, "Graph cut optimization for the piecewise constant level set method applied to multiphase image segmentation," in Scale Space and Variational Methods in Computer Vision. Berlin, Germany: Springer, 2009, vol. 5567, Lecture Notes in Computer Science, pp. 1-13.

[5] C. M. Bishop, Pattern Recognition and Machine Learning (Information Science and Statistics), 2nd ed. Berlin, Germany: Springer, 2007.

[6] A. Blake and M. Isard, Active Contours: The Application of Techniques from Graphics, Vision, Control Theory and Statistics to Visual Tracking of Shapes in Motion, 1st ed. Berlin, Germany: Springer, 2000.

[7] X. Bresson, P. Vandergheynst, and J.-P. Thiran, "A variational model for object segmentation using boundary information and shape prior driven by the Mumford-Shah functional," Int. J. Comput. Vision, vol. 68, pp. 145-162, 2006.

[8] J. Canny, "A computational approach to edge detection," IEEE Trans. Pattern Anal. Mach. Intell., vol. PAMI-8, no. 6, pp. 679-698, Jun. 1986.

[9] A. Chakraborty, L. Staib, and J. Duncan, "Deformable boundary finding in medical images by integrating gradient and region information," IEEE Trans. Med. Imaging, vol. 15, no. 6, pp. 859-870, Dec. 1996.

[10] T. Chan, S. Esedoglu, and K. Ni, "Histogram based segmentation using Wasserstein distances," in Scale Space and Variational Methods in Computer Vision, F. Sgallari, A. Murli, and N. Paragios, Eds. Berlin, Germany: Springer, 2007, vol. 4485, Lecture Notes in Computer Science, pp. 697-708.

[11] T. Chan, S. Esedoglu, and K. Ni, "Histogram based segmentation using Wasserstein distances," in Proc. 1st Int. Conf. Scale Space and Variational Methods in Comput. Vision, 2007, pp. 697-708.

[12] T. Chan and J. Shen, Image Processing And Analysis: Variational, PDE, Wavelet, and Stochastic Methods. Philadelphia, PA, USA: SIAM, 2005.

[13] T. Chan and L. Vese, "Active contours without edges," IEEE Trans. Image Process., vol. 10, no. 2, pp. 266-277, Feb. 2001.

[14] T. Chan and W. Zhu, "Level set based shape prior segmentation," in Proc. IEEE Comput. Soc. Conf. Comput. Vision and Pattern Recognition, 2005, vol. 2, pp. 1164-1170.

[15] L. D. Cohen, "On active contour models and balloons," CVGIP: Image Understanding, vol. 53, no. 2, pp. 211-218, 1991.

[16] D. Cremers, T. Kohlberger, and C. Schn??rr, A. Heyden, G. Sparr, M. Nielsen, and P. Johansen, Eds., "Nonlinear shape statistics in Mumford-Shah based segmentation," in Proc. Computer Vision, 2002, vol. 2351, Lecture Notes in Computer Science, pp. 516-518.

[17] N. El-Zehiry, P. Sahoo, and A. Elmaghraby, "Combinatorial optimization of the piecewise constant Mumford-Shah functional with application to scalar//vector valued and volumetric image segmentation," Image Vision Comput., vol. 29, no. 6, pp. 365-381, 2011.

[18] P. Etyngier, F. Segonne, and R. Keriven, "Shape priors using manifold learning techniques," in Proc. IEEE Int. Conf. Comput. Visionon, 2007, vol. 0 , pp. 1-8.

[19] Y. Fan, T. Jiang, and D. Evans, "Volumetric segmentation of brain images using parallel genetic algorithms," IEEE Trans. Med. Imaging, vol. 21, no. 8, pp. 904-909, Aug. 2002.

[20] I. Figueiredo, P. Figueiredo, G. Stadler, O. Ghattas, and A. Araujo, "Variational image segmentation for endoscopic human colonic aberrant crypt foci," IEEE Trans. Med. Imaging, vol. 29, no. 4, pp. 998-1011, Apr. 2010. 
[21] Y. Freund and R. E. Schapire, "A decision-theoretic generalization of on-line learning and an application to boosting," J. Comput. Syst. Sci., vol. 55, pp. 119-139, Aug. 1997.

[22] K. Fritscher, A. Grunerbl, and R. Schubert, "3d image segmentation using combined shape-intensity prior models," Int. J. Comput. Assisted Radiol. Surgery, vol. 1, pp. 341-350, 2007.

[23] K. Fundana, N. Overgaard, and A. Heyden, "Variational segmentation of image sequences using region-based active contours and deformable shape priors," Int. J. Comput. Vision, vol. 80, pp. 289-299, 2008.

[24] M. Gastaud, M. Barlaud, and G. Aubert, "Combining shape prior and statistical features for active contour segmentation," IEEE Trans. Circuits Syst. Video Technol., vol. 14, no. 5, pp. 726-734, May 2004.

[25] L. Grady and C. Alvino, D. Forsyth, P. Torr, and A. Zisserman, Eds., "Reformulating and optimizing the Mumford-Shah functional on a graph - a faster, lower energy solution," in Computer Vision - ECCV 2008, Berlin, Germany, 2008, vol. 5302, Lecture Notes in Computer Science, pp. 248-261.

[26] R. M. Haralick, K. Shanmugam, and I. Dinstein, "Textural features for image classification," IEEE Trans. Syst., Man Cybern., vol. SMC-3, no. 6, pp. 610-621, Nov. 1973.

[27] T. Hastie and R. Tibshirani, "Classification by pairwise coupling," in Proc. Conf. Adv. Neural Inf. Process. Syst., Cambridge, MA, USA, 1998, vol. 10, NIPS '97, pp. 507-513.

[28] A. Herbulot, S. Jehan-Besson, M. Barlaud, and G. Aubert, "Shape gradient for image segmentation using information theory," in Proc. IEEE Int. Conf. Acoust., Speech, Signal Process., May 2004, vol. 3, pp. iii-21-4.

[29] M. Houghton, J. Lucas, W. Lucas, and J. S. Smith, "A pulsed laser camera system for weld pool observations," in Proc. 16th Int. Conf. Joining of Materials \& 7th Int. Conf. Education in Welding, Tisvildeleje, Denmark, May 2011.

[30] M. Kass, A. Witkin, and D. Terzopoulos, "Snakes: Active contour models," Int. J. Comput. Vision, vol. 1, no. 4, pp. 321-331, 1988.

[31] D. G. Kendall, "A survey of the statistical theory of shape," Statistical Sci., vol. 4, no. 2, pp. 87-99, 1989.

[32] V. Kolmogorov and R. Zabih, "What energy functions can be minimized via graph cuts?," IEEE Trans. Pattern Anal. Mach. Intell., vol. 26, no. 2, pp. 147-159, 2004.

[33] R. Kovacevic and Y. M. Zhang, "Real-time image processing for monitoring of free weld pool surface," J. Manuf. Sci. Eng., vol. 26, no. 119, pp. 161-169, May 1997.

[34] J. Liu, Z. Fan, S. Olsen, K. Christensen, and J. Kristensen, "Towards stabilizing parametric active contours," in Proc. IEEE 4th Annu. Int. Conf. Cyber Technol. in Autom., Control, Intell. Syst., Jun. 2014, pp. 198-203.

[35] Y. Liu, W. Zhang, and Y. Zhang, "Dynamic neuro-fuzzy-based human intelligence modeling and control in GTAW," IEEE Trans. Autom. Sci. Eng., vol. 12, no. 1, pp. 324-335, Jan. 2015.

[36] Y. Liu and Y. Zhang, "Toward welding robot with human knowledge: A remotely-controlled approach," IEEE Trans. Autom. Sci. Eng., vol. 12, no. 2, pp. 769-774, Apr. 2015.

[37] Y. K. Liu and Y. M. Zhang, "Control of 3d weld pool surface," Control Eng. Practice, vol. 21, no. 11, pp. 1469-1480, Nov. 2013.

[38] Y. K. Liu and Y. M. Zhang, "Model-based predictive control of weld penetration in gas tungsten arc welding," IEEE Trans. Control Syst., vol. 22, no. 3, pp. 955-966, May 2014.

[39] X. Ma and Y. M. Zhang, "Reflection of illumination laser from gas metal arc weld pool surface," Meas. Sci. Technol., vol. 20, Jan. 2009, Art. ID 115105.

[40] J. Olivier, R. Bone, J.-J. Rousselle, and H. Cardot, "Active contours driven by supervised binary classifiers for texture segmentation," in Advances in Visual Computing. Berlin, Germany: Springer, 2008, vol. 5358, Lecture Notes in Computer Science, pp. 288-297.

[41] N. Paragios and R. Deriche, "Geodesic active contours and level sets for the detection and tracking of moving objects," IEE Trans. Pattern Anal. Mach. Intell., vol. 22, no. 3, pp. 266-280, Mar. 2000.

[42] N. Paragios and R. Deriche, "Geodesic active regions: A new framework to deal with frame partition problems in computer vision," $J$. Visual Commun. Image Representation, vol. 13, no. 1-2, pp. 249-268, 2002.

[43] N. Paragios and R. Deriche, "Geodesic active regions and level set methods for supervised texture segmentation," Int. J. Comput. Vision, vol. 46, pp. 223-247, Feb. 2002.
[44] Y. Rathi, N. Vaswani, A. Tannenbaum, and A. Yezzi, "Particle filtering for geometric active contours with application to tracking moving and deforming objects," in Proc. IEEE Comput. Soc. Conf. Comput. Vision and Pattern Recognition, Jun. 2005, vol. 2, pp. 2-9.

[45] Y. Shao, Z. Wang, and Y. Zhang, "Monitoring of liquid droplets in laser-enhanced GMAW," Int. J. Adv. Manuf. Technol., vol. 57, no. 1-4, pp. 203-214, 2011.

[46] H.-y. Shen, J. Wu, T. Lin, and S.-b. Chen, "Arc welding robot system with seam tracking and weld pool control based on passive vision," Int. J. Adv. Manuf. Technol., vol. 39, no. 7-8, pp. 669-678, 2008.

[47] Y. Shi, G. Zhang, X. J. Ma, Y. F. Gu, J. K. Huang, and D. Fan, "Laservision-based measurement and analysis of weld pool oscillation frequency in GTAW-P," Welding J., vol. 94, pp. 176s-184s, May 2015.

[48] P. Silapachote, D. R. Karuppiah, and A. R. Hanson, "Feature selection using AdaBoost for face expression recognition," in Proc. IASTED Int. Conf. Visualization, Imaging, and Image Process., Marbella, Spain, Sep. 2004, pp. 84-89.

[49] C. G. Small, The Statistical Theory of Shape. Berlin, Germany: Springer, 1996.

[50] J. S. Smith and C. Balfour, "Real-time top-face vision based control of weld pool size," Ind. Robot: Int. J., vol. 32, no. 4, pp. 334-340, 2005.

[51] H. Song and Y. M. Zhang, "Measurement and analysis of three-dimensional specular gas tungsten arc weld pool surface," Welding J., vol. 87, pp. 85.s-95.s, 2008.

[52] G. Storvik, "A Bayesian approach to dynamic contours through stochastic sampling and simulated annealing," IEEE Trans. Pattern Anal. Mach. Intell., vol. 16, no. 10, pp. 976-986, Oct. 1994.

[53] X. Wang, "Three-dimensional vision-based sensing of GTAW: A review," Int. J. Adv. Manuf. Technol., vol. 72, no. 1-4, pp. 333-345, 2014.

[54] Z. Wang, "Monitoring of GMAW weld pool from the reflected laser lines for real-time control," IEEE Trans. Ind. Inf., vol. 10, no. 4, pp. 2073-2083, Nov. 2014.

[55] Z. Wang, "An imaging and measurement system for robust reconstruction of weld pool during arc welding," IEEE Trans. Ind. Electron., vol. 62 , no. 8, pp. 5109-5118, Aug. 2015.

[56] Z. Wang, X. Ma, and Y. Zhang, "Simultaneous imaging and measurement of pool surface and metal transfer," Welding J., no. $121 \mathrm{~s}-128 \mathrm{~s}$, Jun. 2011

[57] C. S. Wu, J. Q. Gao, X. F. Liu, and Y. H. Zhao, "Vision-based measurement of weld pool geometry in constant-current gas tungsten arc welding," Proc. Inst. Mech. Eng. B, J. Eng. Manufacture, vol. 90, no. 879-882, pp. 669-678, 2003.

[58] C. Xu and J. Prince, "Snakes, shapes, and gradient vector flow," IEEE Trans. Image Process., vol. 7, no. 3, pp. 359-369, Mar. 1998.

[59] C. Xu and J. L. Prince, "Medical image segmentation using deformable models," in Handbook of Medical Imaging-Volume 2: Medical Image Processing and Analysis. Bellingham, WA, USA: SPIE, 2000, vol. 2, pp. 129-174.

[60] N. Xu, N. Ahuja, and R. Bansal, "Object segmentation using graph cuts based active contours," Comput. Vision Image Understanding, vol. 107, no. 3, pp. 210-224, 2007.

[61] A. Yilmaz, X. Li, and M. Shah, "Contour-based object tracking with occlusion handling in video acquired using mobile cameras," IEEE Trans. Pattern Anal. Mach. Intell.., vol. 26, no. 11, pp. 1531-1536, Nov. 2004.

[62] S. C. Zhu and A. Yuille, "Region competition: Unifying snakes, region growing, and Bayes/MDL for multiband image segmentation," IEEE Trans. Pattern Anal. Mach. Intell., vol. 18, pp. 884-900, Sep. 1996.

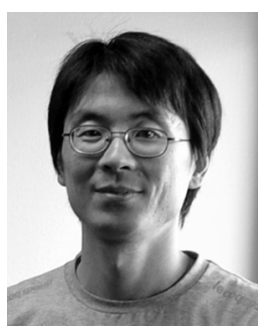

Jinchao Liu received the B.Sc. degree in automation and M.Sc. degree in control science and engineering from the Wuhan University of Technology, Wuhan, China, in 2004 and 2007, respectively, and the Ph.D degree in mechanical engineering from the Technical University of Denmark, Kgs. Lyngby, Denmark, in 2011.

From 2008 to 2011, he was an industrial Ph.D. student at both DTU and FORCE Technology. He is currently the Principal Software Engineer and Scientist at VisionMetric Ltd, Canterbury, U.K. He was an Honorary Research Associate with the School of Physical Sciences, University of Kent, Canterbury, U.K. His current research interests include computer vision, machine learning, vision-based robot control, compressed sensing, and welding automation. 


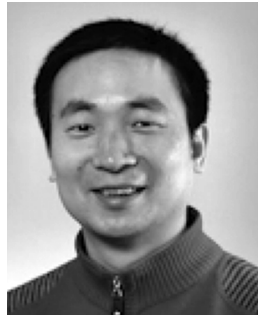

Zhun Fan (SM'14) received the B.S. and M.S. degrees in control engineering from Huazhong University of Science and Technology, Wuhan, China, in 1995 and 2000, respectively, and the Ph.D. degree in electrical and computer engineering from the Michigan State University, Lansing, MI, USA, in 2004.

He is currently a Full Professor with Shantou University (STU), Shantou, China. He also serves as the Head of the Department of Electrical Engineering and the Director of the Guangdong Provincial Key Laboratory of Digital Signal and Image Processing. Before joining STU, he was an Associate Professor with the Technical University of Denmark (DTU) from 2007 to 2011, first with the Department of Mechanical Engineering, then with the Department of Management Engineering, and as an Assistant Professor with the Department of Mechanical Engineering in the same university from 2004 to 2007. He has been a Principle Investigator of a number of projects from Danish Research Agency of Science Technology and Innovation and National Natural Science Foundation of China. His research is also supported by the National Science Foundation. His major research interests include intelligent control and robotic systems, robot vision and cognition, MEMS, computational intelligence, design automation, and optimization of mechatronic systems.

Dr. Fan is the Co-Chair of the Guangzhou Chapter of ACM.

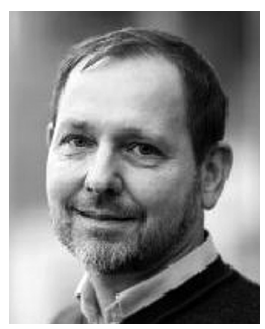

Søren Ingvor Olsen received the Ph.D. degree from the University of Copenhagen, Copenhagen, Denmark, in 1988.

$\mathrm{He}$ is an Associate Professor with The Department of Computer Science, University of Copenhagen, Copenhagen, Denmark. He served as Head of the Department of Computer Science, University of Copenhagen, from 1996 to 1999 . He was member of the Department Government Board from 1993 to 1996 and a member of the Department Educational Board in 2007 and in 2009. He developed the bachelor education in Science and IT (starting at 2010), and serves as Head of Studies. In 2013, he became a member of the educational board for the mathematical and computational studies at SCIENCE. He has participated in a number of projects from The Danish Research Agency of Science Technology and Innovation, The Danish Research Council for Independent Research Natural Sciences, and The Danish Ministry of the Environment. His main research interests include 3-D computer vision, image analysis, robot vision and vision-based automation.

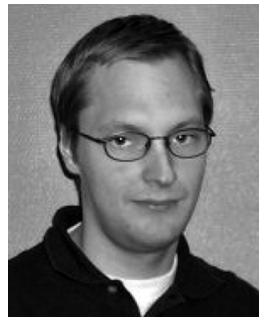

Kim Hardam Christensen received the Ph.D degree from the Technical University of Denmark, Copenhagen, Denmark, in 2004.

He is currently a Project Manager with FORCE Technology (FT), Copenhagen, Denmark. Before joining FT, he was with the Technical University of Denmark, Copenhagen, from 2000 to 2003. Since then he has mainly been working with research and development within the field of welding, especially with modeling, monitoring, control, and optimization of various welding processes.

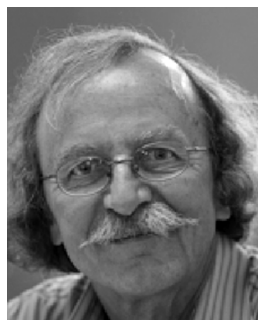

Jens Klæstrup Kristensen is Professor Emeritus (adjunct) in process technology with The Danish Technical University. Many years of experience in working within applied research and technical management within the areas of materials science and process technology, especially laser processing and intelligent automation. For 32 years (1980-2012), he was involved with research and research management at The Danish Welding Institute that now forms a major part of FORCE Technology, Copenhagen, Denmark. He has published 168 scientific papers to date.

Prof. Kristensen is a member of several committees within The International Institute of Welding (IIW) and chairman of Commission IV (Power Beam Processes) 2011-12. Member of the board of the Danish Society for Materials and Testing 1991-98 and again 2004-10. Responsible for organizing several international conferences as e.g., "Advanced Processes and Technologies in Welding and Allied Processes" (2002), "International Conference on Health and Safety in Welding and Allied Processes" (2005) and "Nordic Conference for Laser Processing of Materials - NOLAMP" (1993, 2001 and 2009). He is a member of the Scientific program Committee for several international conferences, e.g., the periodic conferences "Joining of Materials (JOM)" and "Nordic Conference for Laser Processing of Materials (NOLAMP)" and "International Conference on Beam Technology." He was the recipient of the Statoil Prize-93 for outstanding scientific work on process and materials aspects related to laser processing in the heavy industry. He was the recipient of the International Institute of Weldings (IIW's) Houdremont Award in 2002 and the Williams award from the Institute of Materials, Minerals and Mining in 2009. 